[3] C. Goffman and C. J. Neugehauer, On approximate derivatives, Proc. Amer. Math. Soc. II (1960), pp. 962-966.

[4] S. Mareus, On a theorem of Denjoy and on approximate derivatives, Monatsh. Math. 66 (1962), pp. 435-440.

[5] L. E. Snyder, The Baire classification of ordinary and approximate partial derivatives, Proc. Amer. Math. Soc. 17 (1966), pp. 115-123.

[6] G. Tolstoff, Sur quelques propriétés des fonctions approximativement continues, Rec. Math. (Math. Sbornik) N. S. 5 (1939), pp. 637-645.

[7] C. E. Weil, On properties of derivatives, Trans. Amer. Math. Soc. 114 (1965), pp. $363-376$.

[8] Z. Zahorski, Sur la première dérivée, Trans. Amer. Math. Soc. 69 (1950), pp. 1-54.

DEPARTMENT OF MATHEMATICS

BURDWAN UNIVERSITY

Reşu par la Rédaction le 2\%. 11. 1967

\section{One-to-one continuous images of a line}

by

\section{F. Burton Jones (Riverside, Calif.)}

Eren if the metric space $X$ is a one-to-one continuous image of a line, it still may be rather complicated topologically. However, Lelek and McAuley [3] have pointed out that when $X$ is also both locally connected and locally compact, $X$ must be one of five quite simple plane curves: an open interval, a figure eight, a dumbbell, a theta curve, or a noose. In fact, I have indicated in [1] that if $X$ is embeddable in the plane, local compactness is not needed. Consequently one suspects that in the presence of local compactness, local connectivity is too strong and that something weaker should suffice. This turns out to be the case. For if $X$ is aposyndetic and locally compact then $X$ must be one of the five curres. On the other hand, there are plane continuous one-to-one images of the line which are aposyndetic but not locally connected and hence not one of the five curves.

While one of the objects of this paper is to extend the Lelek-McAuley result to the larger class of aposyndetic spaces, it is also one of my purposes to give a more complete argument for the result announced in [1] since the "indication of proof" given there seems not to have been sufficiently suggestive.

Definition. A connected topological space $X$ is aposyndetic at the point $x$ of $X$ provided that if $y$ is a point of $X-x$ there exists a closed and connected set $H$ which contains $x$ in its interior but does not contain $y$ (i.e., $x \in H^{0} \subset H \subset X-y$ ). If $X$ is aposyndetic at each of its points, then $X$ is said to be aposyndetic [2].

THEOREM 1. If the locally compact, aposyndetic, metric space $X$ is a one-to-one continuous image of a line, then $X$ is homeomorphic with an open interval, a figure eight, a dumbbell curve, a theta curve, or a noose (a figure nine).

Proof. Let $f$ denote a one-to-one continuous function from the real numbers onto $X$. Suppose that $U$ is an open subset of $X$ such that (1) $\bar{U}$ is compact and (2) there exist a divergent sequence $t_{1}<t_{2}<t_{3}<\ldots$ of positive numbers and a divergent sequence $t_{-1}>t_{-2}>t_{-3}>\ldots$ of negative numbers such that for each positive integer $n$, both $f\left(t_{n}\right)$ 
and $f\left(t_{-n}\right)$ belong to $X-\bar{U}$. Using Sierpiński's theorem as in [3], each nondegenerate component $C$ of $\bar{U}$ must (for some integer $n$ ) be a subset of $f\left(\left[t_{n}, t_{-n}\right]\right)$. Since $f\left(\left[t_{n}, t_{-n}\right]\right)$ is an are, it can contain at most countably many such components. Consequently the collection $G$ of all nondegenerate components of $\bar{U}$ is countable. Let $\varepsilon$ be a positive number such that for no $g$ in $G$ is $\varepsilon$ the maximum distance from the boundary of $U$ to a point of $g$ but some element of $G$ contains a point whose distance from the boundary of $U$ is more than $\varepsilon$. The subcollection $G_{\varepsilon}$ of $G$ of those elements, each of which contains a point of $U$ at a distance of at least $\varepsilon$ from the boundary of $U$, is an upper semi-continuous collection whose quotient space is compact. Hence at least one element of $G_{\varepsilon}$ fails to contain a limit point of the union of the others. Let $T$ denote such an element. It is clear that $T$ (which is an arc) contains an "open" interval which is open in $X$.

Let $a$ be a point of some such open interval and let $A$ be the union of all open number intervals $I^{0}$ such that $f\left(I^{0}\right)$ is open in $X$ and contains $a$. Let $B=f(A)$.

Case 1. Suppose that $A$ is an open interval of numbers. Then $A$ is maximal with respect to the property of being an open number interval whose image $B$ in $X$ is open in $X$. Suppose that one can find an open subset $R$ of $X$ which contains one end, say $b$, of $B$ (but whose closure does not contain the other end) such that $B \cup R$ is an open set $V$ having the properties: (1) $\bar{R}$ is compact (hence $\bar{V}$ is also compact) and (2) there exist a divergent increasing sequence $\left\{t_{i}\right\}$ of positive numbers and a divergent decreasing sequence $\left\{t_{-i}\right\}$ of negative numbers such that for each positive integer $n$ both $f\left(t_{n}\right)$ and $f\left(t_{-n}\right)$ belong to $X-\bar{V}$. Since this duplicates the conditions imposed on $U$ in the above paragraph it follows that every nondegenerate component of $\bar{V}$ is an arc. Hence the component $C$ of $\bar{V}$ which contains $b$ is an arc containing $b$ as a non-endpoint. Furthermore, since $A$ was chosen to be maximal in the above sense, $b$ is the sequential limit point of a sequence $\left\{b_{i}\right\}$ of points of $R-C$ no two terms of which belong to the same component of $\bar{V}$. For each $i$, let $C_{i}$ denote the component of $\bar{V}$ containing $b_{i}$. Some subsequence of $\left\{C_{i}\right\}$ must converge. So we shall assume that $\left\{C_{i}\right\}$ itself converges to some subarc $Q$ of $C$. Clearly $Q$ cannot contain a point of $B$, but does intersect the boundary of $V$. Let $c$ be a point of $Q$ between $b$ and the boundary of $V$.

Since $X$ is aposyndetic there must exist a closed and connected set $H$ which contains $b$ in its interior but does not contain $c$. Let $W$ denote an open set containing $c$ such that $\bar{W} \subset \nabla-H$. Let $n$ denote a positive integer such that $b_{n}$ belongs to $H$ but such that no subcontinuum of $\bar{V}-W$ contains both $b_{n}$ and a point of the boundary of $\nabla$. Hence $\bar{V}-W$ is the union of two disjoint closed sets containing $b_{n}$ and the boundary of $V$ respectively. Hence $X-W$ is the union of disjoint closed sets doing the same. But $H$ lies in the union of these disjoint closed sets and intersects both of them. This is impossible.

So if at neither end of $B$ can $R$ be found with properties (1) and (2), then each of the sets $\bigcap_{n=1}^{\infty} \operatorname{cl} f(\{t: t \geqslant n\})$ and $\bigcap_{n=1}^{\infty} \operatorname{cl} f(\{t: t \leqslant-n\})$ consists of only one point. Hence it is clear as in Lelek and McAuley's argument that $X$ is either a theta curve or a dumbbell curve.

Case 2. Suppose that $A$ is an-open ray of numbers whose image $B$ in $X$ has end point $b$. If the "unbounded end of $B$ " had two limit points, $X$ would not be aposyndetic. If an open set $R$ can be found which contains $b$ and has properties (1) and (2) of Case 1 , then the argument for Case 1 applies. If no such $R$ exists, then let $A^{\prime}$ be generated by some point $a^{\prime}$ belonging to the complement of $\bar{B}$. If for no such $a^{\prime}$ does this result in Case 1 , then $X$ is a figure eight curre or a noose.

Case 3. Suppose that $A$ is the set of all real numbers. Then clearly $X$ is homeomorphic to an open interval.

The argument is completed by observing that in any open set $U_{0}$ an open subset $U$ of the type postulated at the beginning of the proof must exist. For if $C_{1}, T_{2}$ and $U_{3}$ are all open subsets of $U_{0}$ whose closures are disjoint and compact, then at least one of them enjoys the properties required of $C$.

Exaurle. Let $S$ be the points of

$$
y=(1-x) \sin (\pi / x) \quad(0<x \leqslant 1)
$$

together with the points of

$$
x^{2}+y^{2}=1 \quad(-1<x \leqslant 1 \text { and } y \geqslant 0)
$$

in the $x y$-plane. As a subspace of the plane, $S$ is aposyndetic and a oneto-one continuous image of a line, but $S$ is not homeomorphic to one of the above five curres.

If the metric space $X$ in Theorem 1 is not required to be locally compact but is merely aposyndetic, then even though $X$ is embeddable in the plane it need not be one of the five plane curves. (The space $S$ of the above example demonstrates this fact.) However when aposyndesis is strengthened to local connectivity, the embeddability in the plane produces the desired result.

Plane Images. In the proof of the following lemma use is made of the notion that in $E^{2}$ (or $S^{2}$ ) an arc has two sides. It may be sufficient for the reader to know that if $A_{1}, A_{2}$ and $B$ are three ares in $E^{2}$ such that $A_{i}(i=1,2)$ has only one point in $B$ (and this point is an endpoint of $A_{i}$ and a non-endpoint of $B$ ), and $A_{1}-B$ and $A_{2}-B$ are disjoint, then (1) 
$A_{i}$ is said to abut on $B$ and (2) $A_{1}$ and $A_{2}$ are said to abut on $B$ from the same or different sides according as $h\left(A_{1}-B\right)$ and $h\left(A_{2}-B\right)$ lie on the same or different sides of the $x$-axis where $h$ is a homeomorphism of $E^{2}$ onto itself such that $h(B)$ is a subset of the $x$-axis and $h\left(A_{1}-B\right) \cup h\left(A_{2}-B\right)$ contains no point of the $x$-axis. (In $S^{2}$ one would need to use the equator instead of the $x$-axis.) The elementary and intuitive theorems about abutting ares (e.g., if each of three disjoint arcs abut on an are $B$ then two of them must abut on $B$ from the same side) may be established with the help of well-known theorems in plane topology. For more details the reader is referred to [4].

This theory may be extended in a rather obvious way when certain connected sets replace $A_{1}-B$ and $A_{2}-B$. Suppose that $C$ is a connected subset of $E^{2}-B$ such that $\bar{C} \cap B$ is a subset of an arc $M$ in the interior of $B$. Then if both endpoints of $B$ lie in the same component of $E^{2}-$ $-(\bar{C} \cup M I), C$ is said to abut on $B$. If $C_{1}$ and $C_{2}$ are connected sets each of which abuts on $B$ and $\left(\bar{C}_{1}-B\right) \cap\left(\bar{C}_{2}-B\right)$ are disjoint, then $C_{1}$ and $C_{2}$ would abut on $B$ from different sides if there exists a homeomorphism $h$ of $E^{2}$ onto itself such that $h(B)$ is a subset of the $x$-axis and $h\left(C_{1}\right)$ and $h\left(C_{2}\right)$ are on different sides of the $x$-axis. Equivalently, $C_{1}$ and $C_{2}$ abut on $B$ from different sides if $B$ is a subset of a simple closed curve $J$ such that $C_{1}$ and $C_{2}$ lie in different components of $E^{2}-J$. Similarly, in a fashion analogous to the theory for arcs, one may define the phrase " $C_{1}$ and $C_{2}$ abut on $B$ from the same side" and proceed to establish the elementary theorems.

LEMMA. Suppose that the locally connected metric space $X$ is a oneto-one continuous image of the real numbers. Then if $X$ is embeddable in the 2 -sphere $S^{2}, X$ is locally compact.

Proof. Let $X$ be a subset of $S^{2}$ and let $f$ be a one-to-one continuous mapping of the real numbers onto $X$. If $F$ is a bounded set of numbers, $f \mid F$ is a homeomorphism. And if $V$ is a relatively open subset of $X, f^{-1}(V)$ is an open set of numbers all of whose components except possibly two are open intervals. In general, the topology to be used in this argument (except when referring to number sets) is the topology of the embedding space $S^{2}$ unless otherwise specified. We shall choose some limit point $q$ of $X$ not belonging to $X$ (if there is no such point, $X$ is closed and hence compact) to be used as "the point at infinity." (The reader may find some of the work in Chapter III of [4] useful in following this argument, since we are using an extension of the theory of abutting arcs derived from the work of that chapter and the next.)

Suppose that $D$ is a simple domain in $S^{2}$ which intersects $X$ but whose closure does not contain $q$. Let $V$ denote a component of $D \cap X$. Since $X$ is locally connected, $V$ is open relative to $X$.
Case I. Suppose that there exists an increasing sequence $y_{0}, y_{1}$, $y_{2}, \ldots$ of real numbers such that (1) for each $i, f\left(y_{i}\right) \in S^{2}-\bar{D},(2)\left\{f\left(y_{i}\right)\right\}$ converges to $q$ and (3) $f\left(\left\{t: t \leqslant y_{0}\right\}\right) \subset S^{2}-\bar{D}$. It follows from (2) and (3) that $f^{-1}(V)$ is the union of countably many disjoint open number intervals. Let $\left\{T_{i}\right\}$ denote the set of all images in $X$ of these intervals. Hence $F=\bigcup T_{i}$ such that for each $i, f^{-1}\left(T_{i}\right)$ is an open number interval and $\bar{T}_{i}$ is an are with endpoints in the boundary of $D$.

It is no loss in generality to assume that $f^{-1}(T)$ is not bounded. For if $f^{-1}(V)$ were bounded, $\bar{V}$ would be compact relative to $X$. Hence $\left\{T_{i}\right\}$ is infinite. Since $T$ is connected, each $T_{i}$ must contain a limit point of the union of the others.

We shall first show that for some $T_{i}, V-T_{i}$ has limit points in $T_{i}$ from both sides, i.e., there exist connected open sets $Q_{1}$ and $Q_{2}$ of $V-T_{i}$ which have closures lying in $D$ and which abut on $T_{i}$ from different sides.

Suppose without loss that $f\left(\left[y_{0}, y_{1}\right] \supset T_{1} \cup T_{2} \cup T_{3} \cup T_{4} \cup T_{5}\right.$. There exist connected open subsets $Q_{1}, \ldots, Q_{5}$ of $V-f\left(\left[y_{0}, y_{1}\right]\right)$ whose closures are disjoint and lie in $D$ such that $Q_{1}, \ldots, Q_{5}$ abut on $f\left(\left[y_{0}, y_{1}\right]\right)$ and on $T_{1}, \ldots, T_{5}$ respectively. At least three of them abut on $f\left(\left[y_{0}, y_{1}\right]\right)$ from the same side. For simplicity let us suppose that these are $T_{1}, T_{2}$ and $T_{3}$ and that $f^{-1}\left(T_{1}\right)<f^{-1}\left(T_{2}\right)<f^{-1}\left(T_{3}\right)$. We shall say that $Q_{1}, Q_{2}$ and $Q_{3}$ abut on $T_{1}, T_{2}$ and $T_{3}$ from the same side and call this side the bottom side. (It may help the reader to picture the situation after $E^{2}$ has been transformed by a homeomorphism onto itself which mores $f\left(\left[y_{0}, y_{1}\right]\right.$ into the $x$-axis and $Q_{i}(i=1,2,3)$ into the lower half plane.)

Now let $D_{2}$ be a simple domain such that $\bar{D}_{2} \cap f\left(\left[y_{0}, y_{1}\right]\right)$ is a connected subset of $T_{2}$ containing a point of $\bar{Q}_{2}$ as a non-endpoint, $\bar{D}_{2} \subset D$, and $D_{2}$ is a subset of $D-f\left(\left[y_{0}, y_{1}\right]\right)$ which abuts on $f\left(\left[y_{0}, y_{1}\right]\right)$ from the bottom side. Some component $V_{2}$ of $D_{2} \cap V$ abuts on $f\left(\left[y_{0}, y_{1}\right]\right)$ from the bottom side. Suppose that $y_{2}$ is the first of $y_{2}<y_{3}<\ldots$ such that $f\left(\left[y_{1}, y_{2}\right]\right)$ intersects $V_{2}$. Let $D_{1}$ be a simple domain such that $\bar{D}_{1} \cap f\left(\left[y_{0}, y_{2}\right]\right)$ is a connected subset of $T_{1}$ containing a point of $\bar{Q}_{1}$ as a non-endpoint, $\bar{D}_{1} \subset D-\bar{D}_{2}$, and $D_{1}$ is a subset of $D-f\left(\left[y_{0}, y_{1}\right]\right)$ which abuts on $f\left(\left[y_{0}, y_{2}\right]\right)$ from the bottom side. Some component $V_{1}$ of $D_{1} \cap V$ abuts on $f\left(\left[y_{0}, y_{2}\right]\right)$ from the bottom side. Let $W_{1}$ denote the union of $\bar{V}_{1}$ with all of its complementary domains which lie in $D_{1}$. Clearly $W_{1}$ is a continuum which does not separate $S^{2}$. Let $y_{3}$ be the first of $y_{3}<y_{4}<\ldots$ such that $f\left(\left[y_{0}, y_{3}\right]\right)$ intersects $\nabla_{1}$. Let $a$ and $b$ be numbers $\left(y_{0}<a<y_{1}\right.$ and $\left.y_{1}<b<y_{3}\right)$ such that $f\left(\left[a, y_{1}\right]\right)$ is irreducible from $W_{1}$ to $f\left(y_{1}\right)$ and $f\left(\left[y_{1}, b\right]\right)$ is irreducible from $f\left(y_{1}\right)$ to $W_{1}$. Let $D_{3}$ denote a simple domain such that $\bar{D}_{3} \cap f\left(\left[y_{0}, y_{3}\right]\right)$ is a connected subset of $T_{3}$ containing a point of $\bar{Q}_{3}$ as a non-endpoint, $\bar{D}_{3} \subset D-\left(\bar{D}_{1} \cup \bar{D}_{2}\right)$, and $D_{3}$ is a subset of $D-f\left(\left[y_{0}, y_{3}\right]\right)$ which abuts on $f\left(\left[y_{0}, y_{3}\right]\right)$ from the bottom side. Some component $V_{3}$ of $D_{3} \cap V$ abuts on $f\left(\left[y_{0}, y_{3}\right]\right)$ from the bottom side. 
Now there exists a simple domain $D_{4}$ whose boundary lies in $f\left(\left[y_{1}, b\right]\right) \cup \operatorname{bndy} D_{2}$ such that $D_{4}$ is a subset of $D_{2}-f\left(\left[y_{1}, b\right]\right)$ which abuts on $T_{2}$ from the bottom side and $\bar{D}_{4} \cap f\left(\left[a, y_{1}\right]\right)=\bar{D}_{2} \cap f\left(\left[a, y_{1}\right]\right)$. Some component $V_{4}$ of $\nabla_{2} \cap D_{4}$ abuts on $T_{2}$ from the bottom side and has a limit point $s$ in $f\left(\left[y_{1}, b\right]\right)$. There exists an $n$ sufficiently large such that $f\left(\left[y_{3}, y_{n}\right]\right)$ intersects both $\nabla_{3}$ and $W_{1}$ and hence some subarc $T$ of it is irreducible from $\bar{V}_{3}$ to $W_{1}$. One of the complementary domains $I$ of $f([a, b]) \cup W_{1}$ contains $D_{3} \cup D_{4} \cup\left(T-W_{1}\right)$. In $I \cup f([a, b]) \cup W_{1}$ the point set $W_{1} \cup T \cup \bar{D}_{3}$ separates $\bar{T}_{2}$ from $s$. Hence some subcontinuum $K$ of $T \cap \bar{D}_{4}$ separates $\bar{D}_{4} \frown T_{2}$ from $s$ in $\bar{D}_{4}$. Consequently in $D_{4}-K$ there are connected open subsets of $V_{4}-K$ which abut on $K$ from different sides. But for some number $i, K \subset T_{i}$. Hence there are connected open subsets of $V-T_{i}$ which abut on $T_{i}$ from different sides.

Since $f\left(\left[y_{i}, y_{i+1}\right]\right)(i \geqslant 3)$ covers $V_{3}$ let $\alpha$ denote a simple well-ordering of all those arcs $A$ such that $A$ intersects $V_{3}$ and is the closure of a component of $I \cap f\left(\left[y_{3}, y_{n}\right]\right)$ for some $n>3$ such that both end points of $A$ belong to $W_{1}$. Clearly $I-A$ has' two components, one on the bottom side of $A$ and the other on the other side of $A$.

Since $D_{3}$ has all of the properties postulated for $D$, there is a term of $\alpha$ which has connected open subsets of $V_{3}$ abutting on it from different sides; hence there are terms of $\alpha$ on both sides of it. Since $f^{-1}\left(V_{4}\right)$ has countably many components, let $\mathcal{L}$ be a simple well-ordering of the images in $X$ of these components. Let $A_{0}$ denote the first term of $\alpha$ which has connected open subsets of $V_{3}$ abutting on it from different sides and let $I_{0}$ denote the component of $I-A_{0}$ which does not contain $f([a, b])$ in its boundary. Let $L_{1}$ be the first term of $\mathcal{L}$ in $\bar{I}_{0}$ and let $A_{1}$ be the first term of $\alpha$ lying in $I_{0}-L_{1}$ which has connected open subsets of $V_{3}$ abutting on it from both sides. Let $I_{1}$ denote the component of $I_{0}-A_{1}$ whose closure does not contain $L_{1}$. Now let $L_{2}$ denote the first term of $\mathcal{L}$ lying in $\bar{I}_{1}$ and let $A_{2}$ be the first term of $\alpha$ lying in $I_{1}-L_{2}$ which has connected open subsets of $V_{3}$ abutting on it from both sides. Let $I_{2}$ denote the component of $I_{1}-A_{2}$ whose closure does not contain $L_{2}$. Continue this process. The compact continuum $\bar{D}_{3} \cup\left(\bar{I}_{1} \cap \bar{I}_{2} \cap \bar{I}_{3} \cap \ldots\right) \cup W_{1}$ contains no point of $\bar{V}_{4} \cap f([a, b])$ and yet does not intersect $V_{4}$ which is a contradiction. This completes the proof of Case $I$.

Now let us examine where we stand. If $p$ is a point of $X$ and a simple domain $D$ can be found satisfying conditions (1), (2) and (3) of Case I, then the component $V$ of $D \cap X$ which contains $p$ will have a compact closure relátive to $X$. While conditions (1) and (2) may always be satisfied (one may need to interchange the positive and negative numbers to get $\left\{y_{i}\right\}$ increasing), $p$ could belong to $H^{-}=\bigcap_{n=1}^{\infty} \mathrm{cl} f(\{t: t \leqslant-n\})$ and condition (3) would hold for no choice of $D$. However, if $p$ were the only

point in $H^{-}$, then the argument for Case I can be made to apply with almost no change. So let us assume this to be part of Case I.

Case II. Assume that $\bigcap_{n=1}^{\infty} \mathrm{cl} f(\{t: t \leqslant-n\})$ contains a point of $V$ and a point of $S^{2}-\bar{D}$. More specifically (in addition to the sequence $\left.y_{0}, y_{1}, y_{2}, \ldots\right)$, we shall assume that $\left(3^{\prime}\right)$ there exists a divergent sequence $y_{0}>y_{-1}>y_{-2}>y_{-3}>\ldots$ of real numbers such that for each integer $i$, $f\left(y_{i}\right)$ belongs to $S^{2}-\bar{D}$. Now the argument for Case I applies with only slight changes where $\left\{T_{i}\right\}$ will now be the collection of all sets $T$ such that for some positive integer $n, T$ is a component of $V \cap f\left(\left[y_{-n}, y_{n}\right]\right)$ and in certain other places $\left[y_{-n}, y_{n}\right]$ will be substituted for $\left[y_{0}, y_{n}\right]$.

Since, when $X$ is not locally compact either Case I or Case II would apply, the proof of the lemma is complete.

THEOREM 2. If the locally connected metric space $X$ is a one-to-one continuous plane image of a line, then $X$ is homeomorphic to an open interval, a figure eight, a dumbbell curve, a theta curve, or a noose.

Theorem 2 follows from the above lemma and Theorem 1 of [3] or Theorem 1 above.

Examples in [1] and [3] show that if $X$ is assumed to be embedded in Euclidean 3-space instead of the plane, the conclusion of Theorem 2 need not follow.

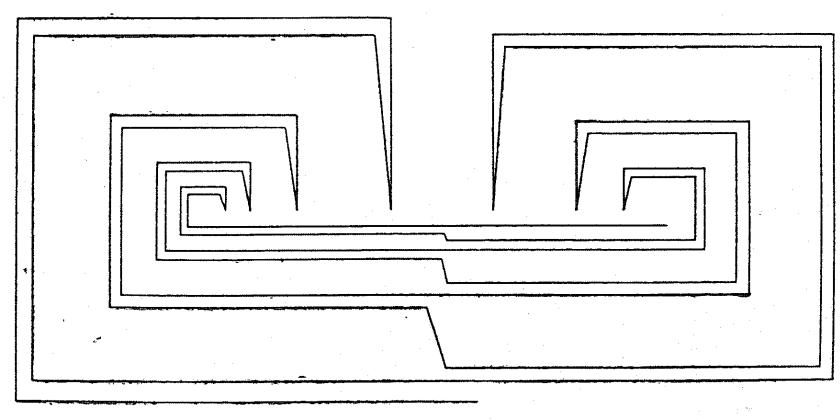

Fig. 1

It is clear that Theorem 2 still holds true if a monotone map is substituted for the one-to-one map. However, finite-to-one maps (or even maps whose point inverses contain no more than two numbers) are not sufficient (see the example in [1]).

It has been pointed out that in Theorem 2 aposyndesis may not be substituted for local connectedness. Since (in the absence of local compactness) semi-local-connectedness is stronger than aposyndesis, one 
may ask is $i t$ strong enough. The answer is "no". The figure above indicates how to construct a one-to-one continuous plane image of a line which is semi-locally-connected but not one of the desired curves.

Question: Can 2-aposyndesis be substituted for local connectivity in Theorem 2? That is, if the one-to-one continuous s-1-c plane image $X$ of a line has the property that for $x$ in $X$ and $y$ and $z$ in $X-x$, there exists a closed (rel. $X$ ) and connected subset of $X-(y+z)$ which contains $x$ in its interior (rel. $X$ ), then is $X$ both locally connected and locally compact?

\section{References}

[I] F. Burton Jones, On the plane one-to-one map of a line, Colloq. Math. 19 (1968), pp. 231-233.

[2] - Concerning aposyndetic and non-aposyndetic continua, Bulletin Amer. Math. Soc. 58 (1952), pp. 137-151.

[3] A. Lelek and L. F. McA uley, On hereditarily locally connected spaces and one-to-one continuous images of a line, Colloq. Math. 17 (1967), pp. 319-324.

[4] R. L. Moore, Foundations of point set theory, Providence 1962.

UNIVERSITY OF CALIFORNIA RIVERSIDE

and THE AUSTRALIAN NATIONAL UNIVERSITY

Reçu par la Rédaction le 13. 5. 1968

\section{Maximal chains in atomic Boolean algebras}

by

\author{
George W. D a y (Laramie, Wyoming)*
}

J. Jakubik [3] has given an example of a Boolean algebra with atoms, which also has a maximal dense-in-itself chain of elements. The principal result of this note is a necessary and sufficient condition that a chain be isomorphic to a maximal chain of an $\mathbf{s}$-complete atomic Boolean algebra. (A Boolean algebra is said to be $\mathbf{s}^{-}$-complete if it is $\mathbf{s}^{\prime}$-complete whenever $s^{\prime}<s$.) In addition, several examples pertinent to related questions on Boolean algebras are given. Our notation will follow that of Dwinger [2].

THEOREM. In order that the chain $C$ be isomorphic to a maximal chain in an $\mathrm{s}^{-}$-complete atomic Boolean algebra, it is necessary and sufficient that $C$ is $\mathrm{N}^{-}$-complete, has a maximal and a minimal element, and has no complete dense-in-itself interval.

Proof. We need only establish that these conditions are sufficient. Let $B$ be the Boolean algebra of finite unions of half-open intervals, $[a, b)$, of $C$. Then $\{[0, c): c \in C\}$ is a maximal chain in $B$, which we hereafter identify with $C$. Let $S(B)$ be the Stone space of $B$, that is, the set of prime ideals of $B$ with the usual topology. Let $P$ be any set of prime ideals of $B$ such that

(i) if $[a, b]$ is a dense-in-itself interval of $C$, then there is an element $I$ of $P$ such that $a+\bar{b} \in I$, and

(ii) if $I \in P$, then $C \cap I$ has no maximal element and $C-I$ has no minimal element.

Note that since $C$ generates $B$, no two elements of $P$ have the same intersection with $C$. Next, let $F$ be the field of sets generated by finite subsets of $P$ and open-and-closed sets in $S(B)$. For each $x \in B$, let $O(x)$ be the corresponding open-and-closed set in $S(B)$; that is, let $O(x)$ $=\{I: I \in S(B)$ and $x \notin I\}$. Denoting symmetric difference by $\oplus$, it is readily shown that $F=\{S \oplus O(x): S$ is a finite subset of $P$ and $x \in B\}$.

LEMTA. $F$ is atomic.

\footnotetext{
* Supported by a University of Wyoming Faculty Summer Research Fellowship.
} 